\title{
Identification of ZBTB26 as a Novel Risk Factor for Congenital Hypothyroidism
}

\author{
Philipp Vick ${ }^{1,+}{ }^{\oplus}$, Birgit Eberle $^{2,+}{ }^{+}$, Daniela Choukair ${ }^{3}$, Birgit Weiss ${ }^{2}$, Ralph Roeth ${ }^{2}$, Isabelle Schneider ${ }^{1}$, \\ Nagarajan Paramasivam ${ }^{4}$, Markus Bettendorf ${ }^{3}$ and Gudrun A. Rappold ${ }^{2, *}$ \\ 1 Department of Zoology, University of Hohenheim, 70599 Stuttgart, Germany; \\ philipp.vick@uni-hohenheim.de (P.V.); isabelle.schneider@uni-hohenheim.de (I.S.) \\ 2 Department of Human Molecular Genetics, Institute of Human Genetics, \\ Ruprecht-Karls-University Heidelberg, 69120 Heidelberg, Germany; Birgit.Koertje@web.de (B.E.); \\ birgit.weiss@med.uni-heidelberg.de (B.W.); ralph.roeth@med.uni-heidelberg.de (R.R.) \\ 3 Division of Paediatric Endocrinology, Children's Hospital, Ruprecht-Karls-University Heidelberg, \\ 69120 Heidelberg, Germany; daniela.choukair@med.uni-heidelberg.de (D.C.); \\ markus.bettendorff@med.uni-heidelberg.de (M.B.) \\ 4 Computational Oncology Group, Molecular Diagnostics Program at the National Center for Tumor \\ Diseases (NCT) and DKFZ (German Cancer Research Center), 69120 Heidelberg, Germany; \\ n.paramasivam@dkfz.de \\ * Correspondence: gudrun_rappold@med.uni-heidelberg.de; Tel.: +49-6221-56-5153 \\ + Shared first authors.
}

Citation: Vick, P.; Eberle, B.; Choukair, D.; Weiss, B.; Roeth, R.; Schneider, I.; Paramasivam, N.; Bettendorf, M.; Rappold, G.A. Identification of ZBTB26 as a Novel Risk Factor for Congenital Hypothyroidism. Genes 2021, 12, 1862. https://doi.org/ $10.3390 /$ genes 12121862

Academic Editor: Paula Soares

Received: 16 September 2021

Accepted: 19 November 2021

Published: 24 November 2021

Publisher's Note: MDPI stays neutral with regard to jurisdictional claims in published maps and institutional affiliations.

Copyright: (C) 2021 by the authors. Licensee MDPI, Basel, Switzerland. This article is an open access article distributed under the terms and conditions of the Creative Commons Attribution (CC BY) license (https:// creativecommons.org/licenses/by/ $4.0 /)$.

\begin{abstract}
Congenital primary hypothyroidism (CH; OMIM 218700) is characterized by an impaired thyroid development, or dyshormonogenesis, and can lead to intellectual disability and growth retardation if untreated. Most of the children with congenital hypothyroidism present thyroid dysgenesis, a developmental anomaly of the thyroid. Various genes have been associated with thyroid dysgenesis, but all known genes together can only explain a small number of cases. To identify novel genetic causes for congenital hypothyroidism, we performed trio whole-exome sequencing in an affected newborn and his unaffected parents. A predicted damaging de novo missense mutation was identified in the ZBTB26 gene (Zinc Finger A and BTB Domain containing 26). An additional cohort screening of 156 individuals with congenital thyroid dysgenesis identified two additional ZBTB26 gene variants of unknown significance. To study the underlying disease mechanism, morpholino knock-down of $z b t b 26$ in Xenopus laevis was carried out, which demonstrated significantly smaller thyroid anlagen in knock-down animals at tadpole stage. Marker genes expressed in thyroid tissue precursors also indicated a specific reduction in the Xenopus ortholog of human Paired-Box-Protein PAX8, a transcription factor required for thyroid development, which could be rescued by adding zbtb26. Pathway and network analysis indicated network links of ZBTB26 to PAX8 and other genes involved in thyroid genesis and function. GWAS associations of ZBTB26 were found with height. Together, our study added a novel genetic risk factor to the list of genes underlying congenital primary hypothyroidism and provides additional support that de novo mutations, together with inherited variants, might contribute to the genetic susceptibility to $\mathrm{CH}$.
\end{abstract}

Keywords: congenital hypothyroidism; thyroid anlagen; Xenopus laevis; thyroid dysgenesis; ZBTB26; PAX8

\section{Introduction}

Congenital primary hypothyroidism (CH; OMIM 218700) is the most common metabolic disorder in newborns with an estimated prevalence of 1 in 2500-3500 births [1,2]. It is characterized by an impaired neurodevelopment and can lead to intellectual disability and growth retardation if untreated. Early diagnosis and therapy are absolutely critical to avoid brain damage. If the serum fT4 concentration is below, and TSH clearly above, 
the age-specific reference interval, then levothyroxine (LT4) treatment should be started immediately [3].

A genetic origin of $\mathrm{CH}$ has been supported by various evidence ([4]; recently reviewed by $[2,5])$. Familial forms of $\mathrm{CH}$ are, however, uncommon. Congenital hypothyroidism can be caused by thyroid dyshormonogenesis or thyroid dysgenesis. Only a minority of children with congenital hypothyroidism present thyroid dyshormonogenesis, while the majority (over $80 \%$ ) present thyroid dysgenesis, a developmental anomaly of the thyroid. In thyroid dysgenesis, different developmental genes including PAX8, FOXE1, NKX2-1, NKX2-5, HHEX, and others have been shown to be affected and heterozygous dominant mutations prevail. All known genes together can only explain a low proportion of the cases [5].

To identify novel genetic causes for congenital hypothyroidism, we performed trio whole-exome sequencing in an affected newborn with high TSH levels together with his unaffected parents. A de novo missense mutation was identified in the ZBTB26 gene (Zinc Finger A and BTB Domain containing 26). Two further ZBTB26 gene variants were identified in a cohort of 156 individuals with congenital hypothyroidism. Pathway and network analysis indicated functional links of ZBTB26 to other known genes underlying thyroid dysgenesis. GWAS studies indicated a significant association with height. Additionally, we report that morpholino knock-down of zbtb26 in X. laevis led to smaller thyroid anlagen and that the Xenopus ortholog of human PAX8 could be rescued by $z b t b 26$, thus giving functional support for the involvement of thyroid dysgenesis.

\section{Materials and Methods}

\subsection{Exome Sequencing and Filtering}

Sequencing was performed using the Illumina HiSeq 2000 system. Raw sequence data were mapped to the 1000 genomes phase II reference genome (GRCh37 version hs37d5) using BWA 0.6.2. Samples showed an average coverage of $94.92 \times$, and $>99 \%$ of bases in the autosomal chromosomes of the target regions have $\geq 10 \times$ coverage and $\geq 20$ QUAL score. SNVs and indels with a read depth of at least $10 \times$ and minimum QUAL score of 20 were considered. Non-synonymous and splice site affecting SNVs and all exonic indels were filtered further. The genotype predicted by Platypus was used to assign genotypes to the variants. Minor allele frequency (MAF) from gnomAD genes and exomes was used and variants with MAF $>0.1 \%$ were removed. In total, 1198 exomes and 3910 WGS samples from the in-house database were used as control to remove common variants and variants present in more than $1 \%$ of the control samples.

\subsection{Sanger Sequencing of Variants}

Thirty-three variants were confirmed by Sanger sequencing. Polymerase chain reaction (PCR) with primers indicated in Supplementary Table S1 was performed with HotStar Taq Polymerase and products analyzed on agarose gels and subsequently sequenced using an ABI machine (Genewiz).

\subsection{Network and Pathway Analysis by Ingenuity Software}

QIAGEN Ingenuity Pathway Analysis (IPA; https:/ / digitalinsights.qiagen.com/, accessed on 15 September 2021) was applied to predict functional connections, and their interpretation was carried out in the context of protein networks that comprise protein-protein interactions and related biological functions as well as canonical signaling pathways.

\subsection{Databases}

TGP (https:/ /browser.1000genomes.org); GnomAD (https:/ / gnomad.broadinstitute. org/, accessed on 15 September 2021); CADD score (https://cadd.gs.washington.edu, accessed on 15 September 2021); dbSNP (https:/ / www.ncbi.nlm.nih.gov, accessed on 15 September 2021); GTEx database (www.gtexportal.org, accessed on 15 September 2021); PROVEAN/SIFT (http:/ / provean.jcvi.org/index.php, accessed on 15 September 2021); 
Polyphen2 (http://genetics.bwh.harvard.edu/pph2/, accessed on 15 September 2021); Mutation Taster (http:/ /www.mutationtaster.org/, accessed on 15 September 2021); GWAS (www.ebi.ac.uk, accessed on 15 September 2021); Disease knowledge portal (https://cvd.hugeamp.org, accessed on 15 September 2021); IPA Ingenuity Systems (https:/ / digitalinsights.qiagen.com/, accessed on 15 September 2021); and NIH GenotypeTissue Expression (GTEx) project. Gene expression of ZBTB26 was accessed in 51 tissues by the NIH Genotype-Tissue Expression (GTEx) database, which is based on RNA-seq data from 8555 tissue samples obtained from 570 adult post-mortem individuals (V6, October 2015).

\subsection{Experimental Approaches with X. laevis}

$X$. laevis care and maintenance, morpholino design, and microinjections, as well as preparation of embryo sections, are given in detail in the Supplementary data.

\subsection{In Situ Hybridization}

A full-length zbtb26 RNA probe was used to perform whole-mount in situ hybridization (ISH). Fixation of embryos was done in MEMFA for $2-3 \mathrm{~h}$ at room temperature. ISH was performed with a Xenopus standard protocol [6]. RNA in situ probes were synthesized with SP6 or T7 polymerases. For tissue-specific analyses of mRNA enrichment after ISH, stained embryos were embedded in $4 \%$ formaldehyde for $3 \mathrm{~h}$ at room temperature. Embryos were embedded in a glutaraldehyde-crosslinked gelatin-albumin mix. Then sectioning was performed with a vibratome (Leica VT1000S; sections $30 \mu \mathrm{m}$ ).

\subsection{Analyses of Thyroid Anlagen}

Thyroid sizes were quantified at stage 45 , when bilateral anlagen were still found in the ventral mesenchyme. Embryos were frontally sectioned and manually screened for thyroids. Equal settings were used to take pictures $(10 \times$ objective of the same microscope, same filter set, same field of view, and same contrast and brightness adjustments). Embryos were only used when their anlagen (or the expected area with remnant tissue) were identified clearly. For individual embryos, the sections with the largest cross-sectional area of the thyroid anlagen were used for each slide [7]. The relative size of both thyroids was determined by measuring the surface area (square pixels) with the ImageJ polygon tool [8]. Then the obtained values were graphically represented as boxplots. Statistical calculations were performed using statistical $R$, with an unpaired Wilcoxon rank sum test with continuity correction (www.R-project.org, accessed on 15 September 2021).

\section{Results}

\subsection{Clinical Data}

Patients included in this study were identified by the newborn screening at the University Children's Hospital Heidelberg. Screening of TSH concentration was measured in dry-blood spots and was carried out by the University of Heidelberg neonatal screening laboratory. Informed consent for exome or targeted sequencing was provided by the respective parents.

Patient 1 with a de novo L75S mutation in ZBTB26 is a eutrophic newborn Caucasian male with $3390 \mathrm{~g}$ birth weight, $56 \mathrm{~cm}$ birth length, and $37 \mathrm{~cm}$ head circumference. Congenital hypothyroidism was detected with a TSH level of $179 \mathrm{mU} / \mathrm{L}$ and normal values for T4, T3, and fT4. Due to the dramatically elevated TSH level, treatment was initiated. Therapy was started with levothyroxine at day 6 and normalization of TSH level was achieved at day 7. No thyroid autoantibodies were detected, and thyroglobulin was initially $159 \mathrm{ng} / \mathrm{mL}$, indicating remaining thyroid tissue. Postnatal ultrasound detected thyroid tissue in the expected position, but no associated malformations. Ultrasound at the age of 9 years, however, detected no thyroid tissues and thyroglobulin was $8.7 \mathrm{ng} / \mathrm{mL}$ while TSH was elevated, indicating thyroid hypoplasia. The boy presented normal intelligence, but dyscalculia and dyslexia at school age. To maintain euthyroidism, increasing dosage of levothyroxine was 
necessary over the time. The family members were Caucasian and non-consanguineous. The grandfather suffered from thyroid nodules, but the younger sister was healthy with normal thyroid function.

Patient 2 with H236R mutation is a eutrophic newborn Caucasian female. Congenital hypothyroidism was detected with a TSH level of $59 \mathrm{mU} / \mathrm{L}$ and reduced values for T4, T3, and fT4. Therapy was started postnatally with levothyroxine, and normalization of TSH level was achieved. The parents reported that they were healthy, but no thyroid examination was performed and they were not available for DNA analysis.

Patient 3 with an intronic variant is a eutrophic Caucasian newborn male, born at 38 gestational weeks with $3500 \mathrm{~g}$ birth weight and $53 \mathrm{~cm}$ birth length. Congenital hypothyroidism was detected with a TSH level $>50 \mathrm{mU} / \mathrm{L}$ and reduced values for T4, T3, and fT4. Therapy was started postnatally with levothyroxine and normalization of TSH level was achieved. Ultrasound at the age of 6 years detected absence of both thyroid and ectopic tissues. There was normal psychomotoric development. To maintain euthyroidism, increasing dosage was necessary over the time. Healthy parents were not available for analysis.

Another patient 332,756 was identified by DECIPHER and presented a $418 \mathrm{~kb}$ deletion encompassing ZBTB26 and 5 additional genes (RC3H2, ZBTB26, RABGAP1, GPR21, and $S T R B P$ ). The patient had a pulmonary stenosis and hypothyroidism, as well as delayed speech and language development. This patient was not included in the analysis as the deleted region contained ZBTB26 as well as an additional 5 genes. Causality of ZBTB could therefore not shown.

\subsection{Exome Sequencing, Filtering and Selection of Candidate Genes}

To identify novel candidate genes implicated in the etiology of congenital hypothyroidism, exome sequencing has been carried out in an affected child and his unaffected parents. Raw sequence data were mapped to the 1000 genomes phase II reference genome (hs37d5) followed by a bioinformatical variant detection pipeline (see Material and Methods). After specific filtering, 650 candidate genes (SNV or Indels) were identified. Further filtering excluded those genes with a minor allele frequency above 0.01 in gnomAD, a consensus score above 1 and a negative intolerance score. Variants were then analyzed for their effect on protein function if they had been predicted as damaging by at least one of the prediction tools (Mutation Taster, Polyphen2, PROVEAN, and SIFT), and with a CADD above 13. A CADD score above 13 indicates that the variant is within the $5 \%$ of the most deleterious substitutions in the human genome. De novo variants were prioritized.

A de novo variant c.224A $>G$ with the top CADD score of 25.4 was identified in the ZBTB26 gene, leading to the amino acid exchange p.L75S (Figure 1A). ZBTB26 (Zink finger and BTB domain -containing protein 26) is a DNA-binding protein regulating transcription. It is a small, highly conserved gene with a transcript conservation between human and Xenopus of 73\% identity. Somatic mutations in ZBTB26 have been identified in different cancers including thyroid carcinoma (https:/ / cancer.sanger.ac.uk/cosmic/gene/analysis? $\ln =$ ZBTB26, accessed on 15 September 2021). ZBTB26 consists of two exons (the first exon is not translated) and a transcript length of 4455 base pairs, encoding a protein of 441 amino acids. The affected Leucine (L) in this individual is highly conserved in mice ( $M$. musculus), rats (R. norvegicus), dogs (Canis), cows (B. taurus), chickens (G. gallus), and frogs (Xenopus), suggesting functional significance. The amino acid change was predicted to be disease-causing/damaging by all the used prediction programs and had previously not been identified in gnomAD (Supplementary Table S1). 


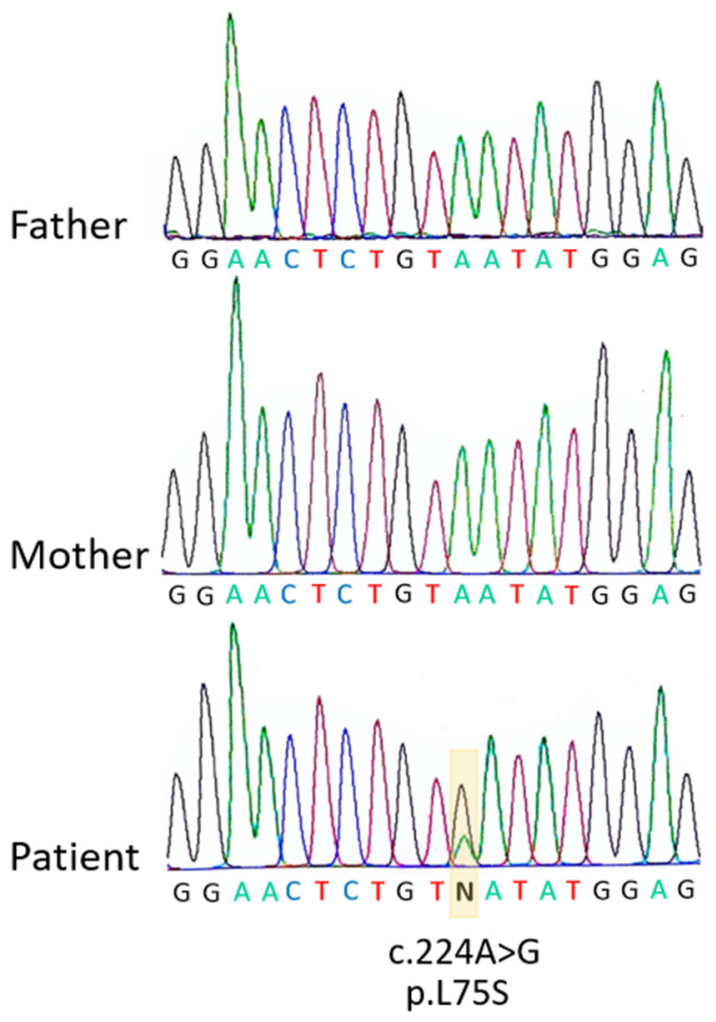

(A)

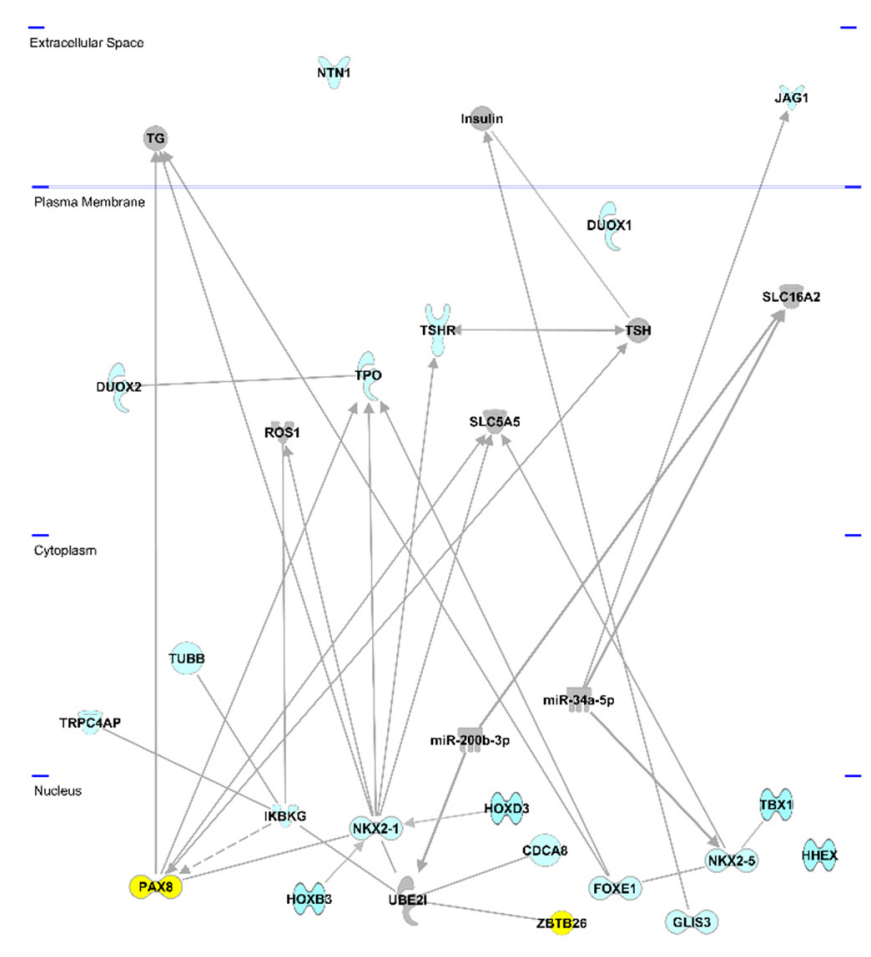

(B)

Figure 1. Identified variants in the ZBTB26 gene and IPA Network analysis with known CH genes. (A) Sequence of trio with unaffected parents and affected child. Sanger sequencing in index case demonstrates a de novo variant $c .224 A>G$ in the ZBTB26 gene which was not present in the unaffected parents. (B) Ingenuity (IPA) Network analysis. Known genes from the literature (in blue color) underlying congenital primary hypothyroidism were analyzed using the IPA network analysis. The newly identified ZBTB26 gene was added to predict functional connections in the context of known protein networks. ZBTB26 and PAX8, both located in the nucleus, are highlighted in yellow. They are connected via NKX2-1 and UBEI. BOREALIN is also called CDCA8.

\subsection{Cohort Screening of 156 Children with Congenital Hypothyroidism for ZBTB26 Variants}

To find out how frequent variants in the ZBTB26 gene are in children with primary hypothyroidism, Sanger Sequence analysis was carried out in an additional cohort of 156 patients with congenital hypothyroidism due to thyroid dysgenesis. Variants in two further individuals were identified, one with a c.707T $>C$ substitution, causing an amino acid exchange p.H236R, and another with an intronic variant, 58 base pairs before the exon-intron junction. DNA of the parents of both patients was not available for analysis. Both variants were predicted to have a CADD score of 17.8 and 17.6. The amino acid change affected a highly conserved amino acid (Histidine 236) between humans and mice (M. musculus), rats (R. norvegicus), dogs (Canis), cows (B. taurus) and chickens (G. gallus). In gnomAD, it was detected in 4 out of 141,406 Europeans, resulting in an allele frequency of 0.00003098. The variant was completely absent in East Asians, but present in 487 Africans, indicating differences among populations. In summary, 2 out of 156 individuals with congenital hypothyroidism presented a risk variant of unknown functional significance in the ZBTB26 gene (Supplementary Table S1).

\subsection{Network Analysis}

To find out if ZBTB26 shares any common functional links with the known genes involved in primary hypothyroidism (indicated in blue color on Figure 1), Ingenuity pathway analysis was carried out. Network links between ZBTB26, UBE2I, NKX2-1, and 
PAX8 could be revealed; all reside in the nucleus (Figure 1B). Protein-protein interactions were identified between ZBTB26 and UBE2I, as well as UBE2I and NKX2-1 [9], and an interaction between PAX8 and NKX2-1 has been described [10]. Together, these data highlight a connection between ZBTB26 and several known $\mathrm{CH}$ genes (Figure 1B).

\subsection{GWAS and Disease Knowledge Databases}

To predict the pathological relevance of the identified ZBTB26 gene in a human phenotype, we consulted the Disease Knowledge Portal (https: / cvd.hugeamp.org, accessed on 15 September 2021) and found GWAS associations of genome-wide significance of several intron variants of CYP26B26 with height (9:125680547_C/T [ $p$-value: $5.83 \times 10^{-14}$ ]; 9:125683121_T/A [ $p$-value: $4.45 \times 10^{-13}$ ]; 9:125689694_C/T [ $p$-value: $1.96 \times 10^{-12}$ ]; and 9:125691119_T/A [ $p$-value: $4.22 \times 10^{-12}$ ]), strongly suggesting that this gene plays a role in final body height. In addition, a genome-wide association was also found with birth weight ( $p$-value: $\left.6.0 \times 10^{-16}\right)$ [11].

\subsection{Experimental Data in X. laevis Provides Functional Support}

As ZBTB26 is expressed in human thyroid gland according to GTEx (among other tissues), we functionally assessed ZBTB26 in thyroid development using the African clawed frog X. laevis as an animal model. Expression of the orthologous zbtb26 gene in the thyroid anlage was shown by in situ hybridization analyses at different stages of tadpole development (tailbud stage 32 to tadpole stage 45 ). Strong signals in the ventral foregut area (Figure $2 \mathrm{~A}, \mathrm{~B}$ ) and in the thyroid precursor were detected (Figure $2 \mathrm{~A}^{\prime}, \mathrm{B}^{\prime}$ ). This was also the case in older tadpole stages, when thyroid anlagen are already split and relocated medially, as well as in the mouth floor epithelium (Figure S1A,B).

Morpholino-mediated knockdown of zbtb26 in the mesendodermal lineage of 4cell stage embryos resulted in a strongly reduced thyroid anlage. We then analyzed expression of known Xenopus orthologs of known CH genes: forkhead box E3 (foxe3), hematopoietically expressed homeobox (hhex), NK2 homeobox 1 (nkx2.1), and paired box 2 (pax2), the amphibian functional ortholog of the mammalian Pax8 gene [12-17]. Our experimental data show that pax 2 was significantly reduced or lost in the ventral thyroid anlagen of zbtb26 morphant embryos (Figure 2C-E), while foxe3, nkx2.1, and hhex expression was left unaltered (Figure $\mathrm{S1C}$ ), suggesting that zbtb26 displays a regulatory role on pax2.

Mid-sagittal sections showed specific reduction in pax 2 in the pre-thyroid tissues of Zbtb26 knockdowns compared to control siblings (Figure 2 $\left.\mathrm{C}^{\prime}, \mathrm{D}^{\prime}\right)$. Further supporting a functional interaction of both genes, sections through the thyroid anlagen of a stage 37 embryo revealed overlapping expression in this area (Figure S1D,E). The effect on pax 2 was specific, as reintroduction of wildtype Xenopus zbtb26 lacking the MO-binding sequence in the $5^{\prime} \mathrm{UTR}$ rescued the pax 2 expression in a significant manner. Interestingly, injecting $z b t b 26$ mRNA alone seemed to have an enhancing or stabilizing effect on pax2 expression as well (Figure 2E).

Next, we wanted to analyze if this reduction in pax 2 expression after zbtb26 loss of function also correlates with a change in thyroid tissue development in early tadpoles. We performed knockdown experiments, either targeting both anlagen or injecting unilaterally into the right or left lineage to hit only one half of the embryo (strictly separated in Xenopus early development), then used the opposite halves as internal controls. When analyzing tadpole frontal sections through the already separated thyroid anlagen at stage 45, we found most morphant thyroids to be reduced in size or not developed at all (Figure 2F-H). Quantification of bilaterally injected specimens revealed an average reduction in size by $36 \%$ in morphant embryos (Figure 2I). Similarly, specimens targeted unilaterally with the $z b t b 26-\mathrm{MO}$ showed about $28 \%$ smaller thyroid anlagen on the injected side than those of the non-injected endogenous control halves (Figure S1F). These results support the conclusion that zbtb26 expressed in the thyroid precursor tissue is required for normal thyroid development. 

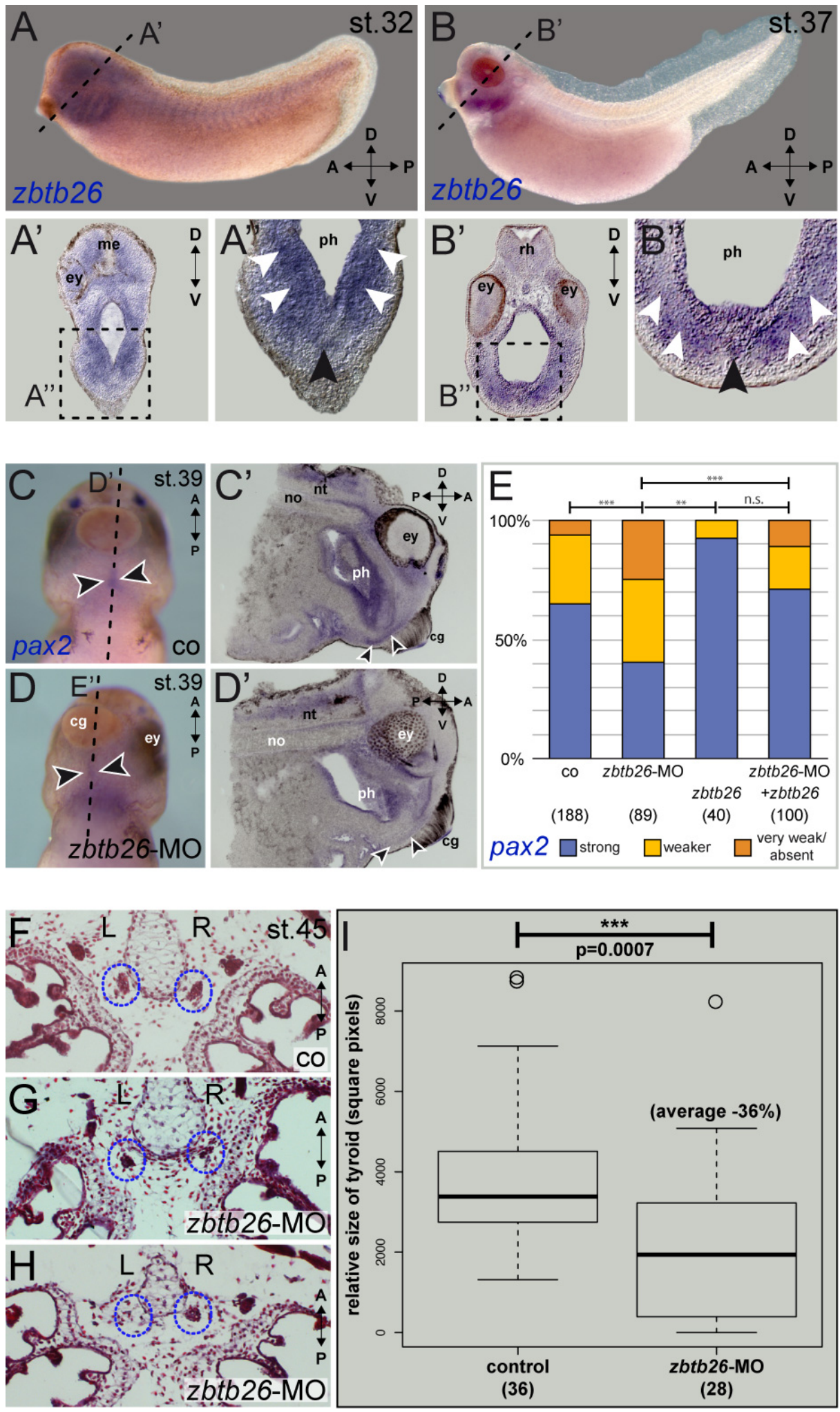

Figure 2. $z b t b 26$ has a conserved role in amphibian thyroid development. (A,B) Developmental expression of $z b t b 26 \mathrm{mRNA}$ in the thyroid anlage of the frog X. laevis. Transcripts in the head are detected ventrally in the medial $\left(\left(\mathbf{A}, \mathbf{B}^{\prime \prime}\right)\right.$, black arrowheads) and ventro-lateral pharyngeal mesenchyme (white arrowheads) of stage $32\left(\mathbf{A}, \mathbf{A}^{\prime \prime}\right)$ and stage $37\left(\mathbf{B}, \mathbf{B}^{\prime \prime}\right)$. Plane of sections in $\left(\mathbf{A}^{\prime}, \mathbf{B}^{\prime}\right)$ are indicated by dashed lines in $(\mathbf{A}, \mathbf{B})$; magnified areas in $\left(\mathbf{A}^{\prime \prime}, \mathbf{B}^{\prime \prime}\right)$ are indicated by dashed boxes in $\left(\mathbf{A}^{\prime}, \mathbf{B}^{\prime}\right)$. (C-E) pax2 expression in the thyroid anlage of stage 39 embryos is reduced by zbtb26 knockdown. Thyroid 
anlage is highlighted by arrowheads. $z b t b 26-\mathrm{MO}$-injected specimens showed reduced or lost expression of pax2 in the thyroid $\left(\mathbf{D}^{\prime}, \mathbf{D}^{\prime}, \mathbf{E}\right)$ compared to control embryos $\left(\mathbf{C}, \mathbf{C}^{\prime}, \mathbf{E}\right)$. This could be rescued by co-injection of full-length $z b t b 26$ mRNA (E). Please note apparently more robust pax2-expression in zbtb26 mRNA-alone injected specimens (E). (F-I) Loss of zbtb26 negatively affects development of the thyroid. Examples of paraffin-sections of control embryo (F) or specimens after knockdown of zbtb26 $(\mathbf{G}, \mathbf{H})$ with paired early thyroids highlighted by blue dotted circles. Please note the strongly reduced right thyroid anlage in $(\mathbf{G})$ and the lost left anlage in $(\mathbf{H})$. Dot plot of quantification of single thyroids showing significantly reduced average size in morphants versus controls (I). A, anterior; $\mathrm{cg}$, cement gland; co, control; D, dorsal; ey, eye; me, mesencephalon; no, notochord; n.s., not significant; nt, neural tube; ph, pharynx; $\mathrm{P}$, posterior; rh, rhombencephalon, $\mathrm{R}$, right; st., stage; and V, ventral. Sample numbers indicated in brackets (in (E), embryos; in (I), left or right side of thyroid anlage). ** $p<0.01,{ }^{* * *} p<0.001$.

\section{Discussion}

Since the identification of the first causal gene for thyroid dysgenesis PAX8 in 1998 [18], a number of genes underlying congenital primary hypothyroidism $(\mathrm{CH})$ have been reported to play a role in this phenotype (e.g., TSHR, NKX2-1, NKX2-5, FOXE1, GLIS3, JAG1, TBX1, NTN1, CDCA8, HOXD3, HOXB3, CDCH8, TUBB1, and TRPC4AP) $[5,19,20]$. These advances have contributed to our understanding of thyroid development and provided very useful information for genetic diagnosis. However, the underlying molecular mechanisms are still poorly understood, and the vast majority of patients and their families are still lacking a genetic diagnosis.

We have provided genetic and functional evidence that ZBTB26 is a further risk gene for this disorder. The ZBTB26 gene is a member of the large ZBTB family of proteins, which are highly conserved transcription factors with essential functions during development (Cheng et al., 2021). All ZBTB family members contain a BTB protein binding and Kruppeltype zink finger DNA binding domain. The BTB domain is used for protein-protein interactions, while the zinc finger domain primarily functions as a DNA binding module. The precise role of most ZBTB family members is still unknown. Several ZBTB proteins have emerged as critical factors during development which regulate the lineage commitment, differentiation and function of lymphoid cells as well as many other developmental processes [21].

In this study, we have identified a de novo missense in ZBTB26 detected in a child with $\mathrm{CH}$, which was not present in his unaffected parents. This child, as well as the other patients analyzed, were all derived from the German newborn screening program. Two additional variants in ZBTB26 were identified in the cohort of 156 children with $\mathrm{CH}$, but as the parents were not available in this study, it remains unsolved if these variants occurred de novo or not. As the detected ZBTB26 variants were rare in this cohort (2/156), a high genetic heterogeneity is presumed, which is in line with former investigations.

An oligogenic model of inheritance for $\mathrm{CH}$ has also been proposed by previous studies, possibly together with a modulation by environmental modifiers $[5,22]$. This may also apply to our patients, at least to the patient, where exon sequencing has been performed. In this patient with the de novo ZBTB26 mutation, further weak variants in additional known $\mathrm{CH}$ genes (PAX8, TUBB1) were transmitted from either parent to the child (the $P A X 8$ variant from mother; the TUBB1 variant from father), suggesting that a combination of different variants would be needed to lead to the full-blown phenotype (Supplementary Table S2). The co-occurrence of de novo and familiar mutations in genes involved in $\mathrm{CH}$ also highlight that epistasis between different specific gene loci can modulate the risk for $\mathrm{CH}$. Different susceptibility variants for $\mathrm{CH}$ variants may thus act together, and in some cases, additional epigenetic or environmental factors may be also necessary for the emergence of the disorder. This oligogenic model of inheritance or multi-hit model for $\mathrm{CH}$ has been already proposed before [5,22] and may also apply to our patients.

Finally, functional studies have shown that ZBTB26 represents a convincing risk gene for $\mathrm{CH}$. Network analysis has shown that ZBTB26 is linked to several known $\mathrm{CH}$ genes 
(CDCA8, NKX2-1, and IKBKG) via UBE2I, and according to the GTEX database, ZBTB26 has one of its highest expressions in the thyroid. In Xenopus, which was used as an animal model, $z b t b 26$ transcripts were found in the early thyroid anlagen and continued to be active in early thyroids at different stages of tadpole development (Figures 2 and S1A,B). Gene activity can easily be manipulated in Xenopus by microinjection into its large early embryos right after fertilization. These knockdown experiments demonstrated significantly smaller thyroid anlagen and smaller thyroids at the tadpole stage, thus indicating a direct effect of its reduced expression.

In summary, a causal link of $Z B T B 26$ variants and $C H$ is supported by several lines of evidence: Variants with evolutionary conservation of affected amino acids and good algorithm predictions with an effect on protein function and low frequency in the general Caucasian population were detected in altogether 3/157 individuals with $\mathrm{CH}$. In addition, reported associations with final height and the gene expression patterns in the thyroid in human and frog supported these findings. Finally, the regulatory role on pax 2 expression, and the in vivo requirement for early thyroid organogenesis in tadpole furthermore speak for a relevance of this gene in $\mathrm{CH}$. Our study also provides additional support that de novo mutations, together with inherited variants, might contribute to the genetic susceptibility to $\mathrm{CH}$. To date, it is not clear how many genes contribute to the phenotype of $\mathrm{CH}$ and how these different variants interact with each other. A better knowledge of these complex interactions will be needed to fully understand this common endocrine disorder.

Supplementary Materials: The following are available online at https:/ / www.mdpi.com/article/10 .3390/genes12121862/s1: Table S1: Identified ZBTB variants in patients with congenital hypothyroidism, Table S2: Additional variants in known hypothyroid genes and gnomAD analysis of variants compared with ZBTB26, Figure S1: zbtb26 has a conserved role in amphibian thyroid development; Supplementary data: Xenopus laevis: care and maintenance, morpholino design, microinjection and embryo sections.

Author Contributions: Conceptualization, M.B. and G.A.R.; methodology, B.E., B.W. and P.V.; software, B.W. and N.P.; validation, P.V. and B.W.; resources, D.C. and M.B.; data curation, B.E., P.V., I.S., R.R. and B.W.; writing-original draft preparation, P.V., B.E. and D.C.; writing—review and editing, P.V., D.C., B.E. and G.A.R.; supervision, G.A.R.; funding acquisition, G.A.R. and M.B. All authors have read and agreed to the published version of the manuscript.

Funding: This research was funded by the Medical Faculty of the University of Heidelberg.

Institutional Review Board Statement: The study was conducted according to the guidelines of the Declaration of Helsinki and approved by the Ethical Committee of the University Hospital Heidelberg (S-636/2012).

Informed Consent Statement: Informed consent was obtained from all subjects involved in the study.

Data Availability Statement: All data can be obtained by the authors.

Acknowledgments: We thank A. Schäfer-Kosulja and Heinz Streble for expert technical advice and assistance with embryo sectioning, and Verena Andre and Sebastian Vogel for general technical assistance. We also thank Martin Blum for initiating the contact to the Hohenheim lab, the DKFZ Omics IT and Data Management Core Facility (ODCF), and the DKFZ Genomics and Proteomics Core Facility (GPCF) for excellent technical support.

Conflicts of Interest: The authors declare no conflict of interest.

\section{References}

1. Bettendorf, M. Thyroid disorders in children from birth to adolescence. Eur. J. Nucl. Med. Mol. Imaging 2002, 29 (Suppl. S2), S439-S446. [CrossRef]

2. Stoupa, A.; Kariyawasam, D.; Carre, A.; Polak, M. Update of Thyroid Developmental Genes. Endocrinol. Metab. Clin. N. Am. 2016, 45, 243-254. [CrossRef] [PubMed] 
3. Trotsenburg, P.; Stoupa, A.; Leger, J.; Rohrer, T.; Peters, C.; Fugazzola, L.; Cassio, A.; Heinrichs, C.; Beauloye, V.; Pohlenz, J.; et al. Congenital Hypothyroidism: A 2020-2021 Consensus Guidelines Update-An ENDO-European Reference Network Initiative Endorsed by the European Society for Pediatric Endocrinology and the European Society for Endocrinology. Thyroid 2021, 31, 387-419. [CrossRef]

4. Bernheim, M.; Berger, M.; Uzan, R. Le rôle des facteurs génétiques dans le développement du myxoedème congénital [The role of genetic factors in the development of congenital myxedema]. C. R. Seances Soc. Biol. Fil. 1955, 149, 1553-1558. [PubMed]

5. Stoupa, A.; Kariyawasam, D.; Muzza, M.; de Filippis, T.; Fugazzola, L.; Polak, M.; Persani, L.; Carre, A. New genetics in congenital hypothyroidism. Endocrine 2021, 71, 696-705. [CrossRef]

6. Sive, H.L.; Grainger, R.M.; Harland, R.M. Early Development of Xenopus Laevis; CSHL Press: Cold Spring Harbor, NY, USA, 2000.

7. Grim, K.C.; Wolfe, M.; Braunbeck, T.; Iguchi, T.; Ohta, Y.; Tooi, O.; Touart, L.; Wolf, D.C.; Tietge, J. Thyroid histopathology assessments for the amphibian metamorphosis assay to detect thyroid-active substances. Toxicol. Pathol. 2009, 37, 415-424. [CrossRef] [PubMed]

8. Schneider, C.A.; Rasband, W.S.; Eliceiri, K.W. NIH Image to ImageJ: 25 years of image analysis. Nat. Methods 2012, 9, 671-675. [CrossRef]

9. Rolland, T.; Tasan, M.; Charloteaux, B.; Pevzner, S.J.; Zhong, Q.; Sahni, N.; Yi, S.; Lemmens, I.; Fontanillo, C.; Mosca, R.; et al. A proteome-scale map of the human interactome network. Cell 2014, 159, 1212-1226. [CrossRef]

10. Ravasi, T.; Suzuki, H.; Cannistraci, C.V.; Katayama, S.; Bajic, V.B.; Tan, K.; Akalin, A.; Schmeier, S.; Kanamori-Katayama, M.; Bertin, N.; et al. An atlas of combinatorial transcriptional regulation in mouse and man. Cell 2010, 140, 744-752. [CrossRef]

11. Warrington, N.M.; Beaumont, R.N.; Horikoshi, M.; Day, F.R.; Helgeland, O.; Laurin, C.; Bacelis, J.; Peng, S.; Hao, K.; Feenstra, B.; et al. Maternal and fetal genetic effects on birth weight and their relevance to cardio-metabolic risk factors. Nat. Genet. 2019, 51, 804-814. [CrossRef]

12. Heller, N.; Brandli, A.W. Xenopus Pax-2/5/8 orthologues: Novel insights into Pax gene evolution and identification of Pax-8 as the earliest marker for otic and pronephric cell lineages. Dev. Genet. 1999, 24, 208-219. [CrossRef]

13. Hollemann, T.; Pieler, T. Xnkx-2.1: A homeobox gene expressed during early forebrain, lung and thyroid development in Xenopus laevis. Dev. Genes Evol. 2000, 210, 579-581. [CrossRef]

14. El-Hodiri, H.M.; Seufert, D.W.; Nekkalapudi, S.; Prescott, N.L.; Kelly, L.E.; Jamrich, M. Xenopus laevis FoxE1 is primarily expressed in the developing pituitary and thyroid. Int. J. Dev. Biol. 2005, 49, 881-884. [CrossRef]

15. Zorn, A.M.; Wells, J.M. Vertebrate endoderm development and organ formation. Annu. Rev. Cell Dev. Biol. 2009, 25, $221-251$. [CrossRef] [PubMed]

16. Rankin, S.A.; Kormish, J.; Kofron, M.; Jegga, A.; Zorn, A.M. A gene regulatory network controlling hhex transcription in the anterior endoderm of the organizer. Dev. Biol. 2011, 351, 297-310. [CrossRef] [PubMed]

17. Wang, J.H.; Deimling, S.J.; D’Alessandro, N.E.; Zhao, L.; Possmayer, F.; Drysdale, T.A. Retinoic acid is a key regulatory switch determining the difference between lung and thyroid fates in Xenopus laevis. BMC Dev. Biol. 2011, 11, 75. [CrossRef]

18. Macchia, P.E.; Lapi, P.; Krude, H.; Pirro, M.T.; Missero, C.; Chiovato, L.; Souabni, A.; Baserga, M.; Tassi, V.; Pinchera, A.; et al. PAX8 mutations associated with congenital hypothyroidism caused by thyroid dysgenesis. Nat. Genet. 1998, 19, 83-86. [CrossRef]

19. Carre, A.; Stoupa, A.; Kariyawasam, D.; Gueriouz, M.; Ramond, C.; Monus, T.; Leger, J.; Gaujoux, S.; Sebag, F.; Glaser, N.; et al. Mutations in BOREALIN cause thyroid dysgenesis. Hum. Mol. Genet. 2017, 26, 599-610. [CrossRef]

20. Choukair, D.; Eberle, B.; Vick, P.; Hermanns, P.; Weiss, B.; Paramasivam, N.; Schlesner, M.; Lornsen, K.; Roeth, R.; Klutmann, C.; et al. Identification of Transient Receptor Potential Channel 4-Associated Protein as a Novel Candidate Gene Causing Congenital Primary Hypothyroidism. Horm. Res. Paediatr. 2020, 93, 16-29. [CrossRef]

21. Cheng, Z.Y.; He, T.T.; Gao, X.M.; Zhao, Y.; Wang, J. ZBTB Transcription Factors: Key Regulators of the Development, Differentiation and Effector Function of T Cells. Front. Immunol. 2021, 12, 713294. [CrossRef] [PubMed]

22. Amendola, E.; De Luca, P.; Macchia, P.E.; Terracciano, D.; Rosica, A.; Chiappetta, G.; Kimura, S.; Mansouri, A.; Affuso, A.; Arra, C.; et al. A mouse model demonstrates a multigenic origin of congenital hypothyroidism. Endocrinology 2005, 146, 5038-5047. [CrossRef] [PubMed] 